\title{
El docente multitarea en L2. Enseñanza intergeneracional, intercultural y multimodal
}

MARÍA JesÚs LlORENTE PUERTA

Accem

mllorentep@accem.es

\begin{abstract}
Resumen: La oenegé Accem lleva a cabo un proyecto denominado Siempre hay tiempo: un programa de conciliación de la vida formativa y personal. Herramientas para la residencia legal y la ciudadanía activa. La actividad principal estriba en la enseñanza del español como segunda lengua (EL2) a alumnado inmigrante adulto. En el presente artículo pretendemos mostrar que una enseñanza enfocada a tales destinatarios ha de ser multimodal, intergeneracional e intercultural y requiere de un profesorado multitarea cuya práctica docente se adapte a las necesidades y a la vida de nuestro alumnado.
\end{abstract}

Palabras clave: EL2, alumnado inmigrante, integración, ciudadanía.

The multitasking teacher in L2. Intergenerational, intercultural and multimodal teaching.

Abstract: The NGO Accem is carrying out a project called There is always time: a reconciliation between personal life and learning program. Tools for legal residence and active citizenship. The main activity lies in the teaching of Spanish as a second language (EL2) to adult immigrant students. With this work we aim to reflect that a teaching that targets on these apprentices must be multimodal, intergenerational and intercultural and requires a multitasking teachers whose teaching practice must be adapted to the needs and life of our students.

Key words: SL2, immigrant students, integration, citizenship.

\section{Introducción.}

El objetivo de este trabajo es presentar un proyecto concreto llamado Siempre hay tiempo. Programa de conciliación de la vida formativa y personal. Herramientas para la residencia legal y la ciudadanía activa. Se trata de un programa de la ONG Accem ${ }^{1}$ que tiene como actividad principal la enseñanza del español como segunda lengua, está financiado por el Ministerio de Inclusión, Seguridad Social y Migraciones y el Fondo de Asilo, Migración e Integración y se dirige a personas nacionales de terceros países con residencia en España, esto es, a personas inmigrantes.

En este año 2020 se cumple el 25 aniversario de las primeras jornadas acerca de la enseñanza del español con personas inmigrantes y refugiadas que organizó el Departamento de Didáctica de la Lengua y la Literatura de la Universidad Complutense de Madrid en $1995^{2}$. Se puede considerar que ya existe una trayectoria de peso y que es

1 www.accem.es

2 Las actas completas de las jornadas pueden consultarse en https://dialnet.unirioja.es/ejemplar/14818

Foro de Profesores de E/LE, número 16 (2020) 
este campo, el de la enseñanza del español a alumnado inmigrante, uno de los más dinámicos en el ámbito de la didáctica del español como segunda lengua, pues cada vez es mayor la organización de congresos, mesas de experiencias, debates, ponencias y comunicaciones en entornos académicos (Pastor, 2007; Villalba, 2012; Llorente, 2018). Por lo general, la expresión español para inmigrantes remite a cierto imaginario que poco tiene que ver con la enseñanza de lenguas y mucho con la consideración socioeconómica del alumnado (Villalba y Hernández, 2010). Frecuentemente se atribuye a las personas inmigrantes cierto déficit cultural e incluso cognitivo, pues existe el prejuicio que asocia conocimientos y habilidades de las personas con su condición económica y social (Esteban, 2011). Nosotros partimos del convencimiento de que el hecho de ser inmigrante no constituye un elemento decisivo en el proceso de aprendizaje del español como segunda lengua. No obstante, como señala el Instituto Cervantes en su Curso de emergencia destinado a inmigrantes (Sanz, 2005), este alumnado puede tener que enfrentarse a diferencias sociales debidas a la existencia de prejuicios y estereotipos, a desigualdades relativas al género o el estatus, a la diversidad de niveles educativos y también a dificultades personales relacionadas con la carencia de redes de apoyo y con problemas para conciliar la vida formativa y laboral con la personal y familiar. Somos conscientes de las particularidades de este grupo que, además de necesitar el dominio de la lengua de la sociedad de acogida debe enfrentarse a una serie de desafíos más allá de las diferencias culturales relacionadas con los distintos valores y las diversas prioridades y tradiciones.

\section{La enseñanza intergeneracional, intercultural y multimodal.}

Los calificativos de intergeneracional, intercultural y multimodal, además de ser válidos en múltiples escenarios de enseñanza de segundas lenguas, dibujan la situación del alumnado inmigrante. Esos tres adjetivos pueden aplicarse a nuestras aulas, donde todos los estudiantes entran en una categoría que se ajusta a ellos: la heterogeneidad. Personas diversas que se acercan espontáneamente a la asociación atraídas por la oferta formativa, por las recomendaciones de familiares o conocidos, o derivadas por otras entidades o instituciones.

En primer lugar, la enseñanza es intergeneracional porque en ella participan alumnos de todas las edades. Existen grupos específicos de menores no acompañados, jóvenes extranjeros entre los 16 y los 18 años que se encuentran en España sin sus padres, tutelados por las administraciones. También hay personas mayores, abuelos que han acompañado a sus hijos en el proyecto migratorio o que han venido después, una vez que sus familias se han asentado en España. No obstante, lo habitual es que los grupos se organicen por nivel de dominio del español, no por edad. Las clases de adultos incluyen personas de los 16 a los 76 años, menores adolescentes y jubilados que interactúan en el aula trabajando colaborativamente en un aprendizaje entre iguales.

En segundo lugar, se trata de enseñanza intercultural que se dirige a aprendices de todas las procedencias con lenguas, costumbres, religiones y prácticas variadas, con experiencias diversas y diferentes esquemas de valores. Alumnado que se encuentra en situaciones personales más o menos complejas y posee múltiples expectativas y objetivos de aprendizaje. Como señala el Marco común europeo de referencia para las lenguas: aprendizaje, enseñanza y evaluación (MCER) (Consejo de Europa, 2002), la enseñanza trasciende el tradicional enfoque de la competencia comunicativa y se acerca 
a la competencia plurilingüe y pluricultural. Se ha de desarrollar en el alumnado una competencia intercultural que el Plan Curricular del Instituto Cervantes (Instituto Cervantes, 2006) define como la capacidad del alumnado para adaptarse y desenvolverse con éxito en distintas situaciones sociales en las que se relaciona con personas de comunidades distintas a la propia o analiza hechos y productos culturales característicos de estas comunidades.

Para adaptarse a esa heterogeneidad se impone el empleo de variedad de métodos, actividades y recursos. La enseñanza ha de ser, por tanto, necesariamente multimodal, entendida como la construcción de significados por medio de diferentes modos de expresión (Kress, 2010; Berrios, 2018) y tiene que estar planificada de una manera reflexiva y coherente. La metodología combina opciones didácticas y el uso de nuevas tecnologías que apoyan el aprendizaje o trasladan su entorno físico a uno virtual. Es aquí donde lo multimodal cobra pleno sentido pues se integran diversos modos de representación del conocimiento (audios, lenguaje oral, escrito, imagen fija y en movimiento, infografías, etc.) en un único formato multimedia (Cassany, 2003).

\section{El docente multitarea.}

La enseñanza de EL2 sirve como herramienta para la adquisición y mantenimiento de la residencia legal en España de las personas extranjeras y para facilitar el ejercicio de la ciudadanía activa.

En primer lugar, el paso previo a toda planificación educativa en enseñanza de segundas lenguas pasa por un análisis de las necesidades e intereses de los aprendices (Richards y Rogers, 2002; Woodward, 2002). En nuestro caso, la programación de las actividades formativas la lleva a cabo el profesorado y se basa en la observación directa de los grupos, en el análisis de la información de grupos de discusión organizados ad hoc y en pruebas de nivel estandarizadas, según los casos.

Nuestro método de enseñanza y aprendizaje del español toma para la detección de necesidades de comunicación la propuesta de Maslow (1943) (imagen 1) sobre la jerarquía de necesidades humanas:

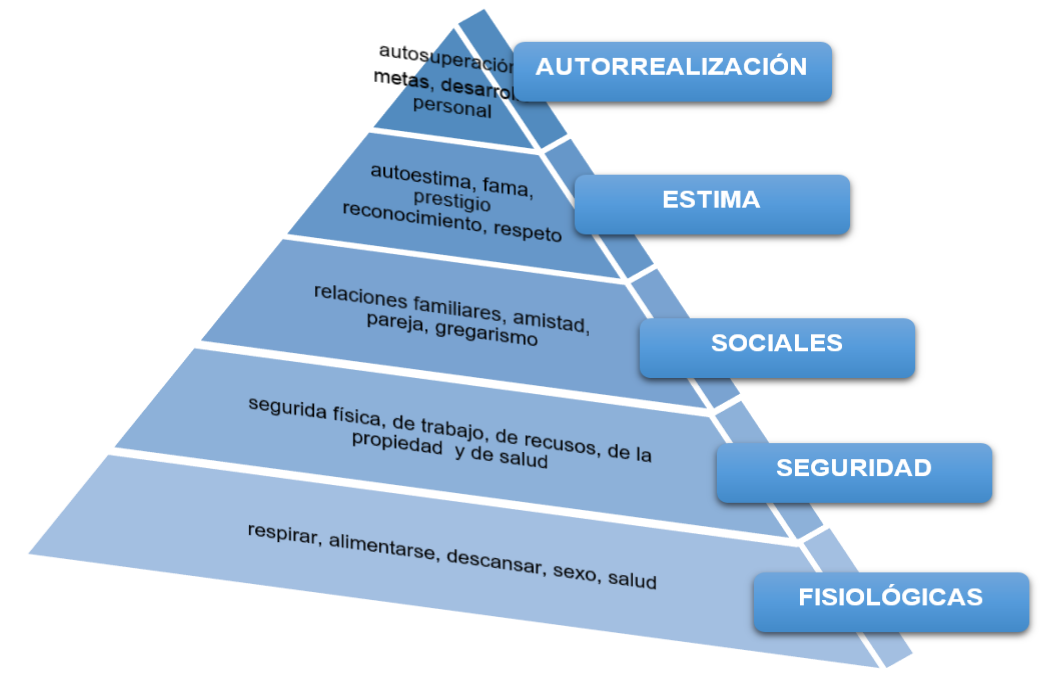


Entre esas necesidades que van de las más básicas y de supervivencia a las más subjetivas, dentro de lo que puede ser cada expectativa individual, una característica común del alumnado es su intención de vivir en España. En la motivación hacia el aprendizaje del español como segunda lengua entran en juego los fines instrumentales, como puede ser la búsqueda de empleo o la finalidad integrativa, el deseo de formar parte de la comunidad (Gardner y Lambert, 1972).

En segundo lugar, según esas necesidades, se diseñan actividades que se corresponden con el objetivo principal del proyecto: «Utilizar la formación lingüística y socioeducativa para impulsar la ciudadanía activa entre el colectivo inmigrante y promover el diálogo intercultural a través de formaciones que faciliten la conciliación entre la vida laboral, formativa, personal y familiar de las personas que participan en el proyecto».

Y en este contexto, un elemento fundamental es la figura del profesorado. La sensibilidad hacia el colectivo resulta un requisito imprescindible, pero también su formación, su experiencia y, en el caso concreto de este proyecto, donde se ha de servir de las TIC para el desarrollo del trabajo, la competencia digital docente (INTEF, 2017) se presupone. Los profesores deben poseer todas y cada una de las competencias que señala el Instituto Cervantes (2012): además de participar activamente en la institución que promueve los cursos, organizar situaciones de aprendizaje, implicar al alumnado en el proceso y evaluar, debe facilitar la comunicación intercultural, desarrollarse profesionalmente, luchando contra los estereotipos que rodean a esta actividad y ha de gestionar de manera especial los sentimientos y emociones en su trabajo y las expectativas que pueda tener a la hora de acercarse a esta actividad.

En tercer lugar, en consonancia con el objetivo del proyecto y el perfil del profesorado, impartimos cursos de español como segunda lengua que inciden en contenidos socioculturales y acercan al alumnado al conocimiento de la lengua, la sociedad y la cultura españolas.

\subsection{Metodología.}

Tres son las modalidades de formación que se ofrecen y que hacen referencia a la simultaneidad o no de docentes y aprendices en el mismo espacio físico: presencial, semipresencial y a distancia y en todas ellas se trabajan contenidos lingüísticos y culturales y talleres y módulos específicos vinculados a la legislación, la sanidad, la educación, la perspectiva de género, la sensibilidad medioambiental, la vivienda, los recursos comunitarios, la participación social y el empleo en la nueva sociedad.

La formación se concreta en:

- cursos de español como segunda lengua en diferentes niveles. Los niveles de formación en lengua y cultura españolas se adaptan a la política lingüística y educativa, en nuestro caso al Marco común europeo de referencia para las lenguas: aprendizaje, enseñanza y evaluación (Consejo de Europa, 2002), y los ámbitos de uso que en él aparecen reflejados. Las actividades lingüísticas se articulan en torno a varios ejes temáticos: Las presentaciones y la identificación personal; El entorno y la ciudad en la que vivo; El consumo y 
las compras; Sanidad: salud y estado físico; La vivienda; El trabajo; El ocio y el tiempo libre.

- talleres específicos de contenido sociocultural, como la organización política y territorial de España, el cuidado del medioambiente, la igualdad de oportunidades entre hombres y mujeres, la perspectiva de género o el ejercicio de la participación social y la ciudadanía activa.

- cursos para preparar el examen de conocimiento constitucional y sociocultural de España, necesario para obtener la nacionalidad española.

El contexto de enseñanza y aprendizaje determina la opción metodológica. Puesto que se trata de un aprendizaje del español como segunda lengua en situación de inmersión, la finalidad es práctica. Esto se refiere a «un contexto de uso y aprendizaje de la nueva lengua por lo que cualquier enfoque metodológico que se suscriba debe fundamentarse en una perspectiva centrada en la acción» (Ríos Rojas, 2016:18).

En la enseñanza presencial las actividades tienen como público diana a aquellas personas con especial dificultad para conciliar su vida personal con la formativa. Así, una de las clases más demandadas en la que denominamos informalmente grupos de mamás (imagen 2) puesto que están conformadas por mujeres que acuden a las clases de español como segunda lengua acompañadas de sus criaturas menores de tres años. Se trata de personas que, por diversos motivos, no han podido acceder a dispositivos de escolarización de primer ciclo de educación infantil.

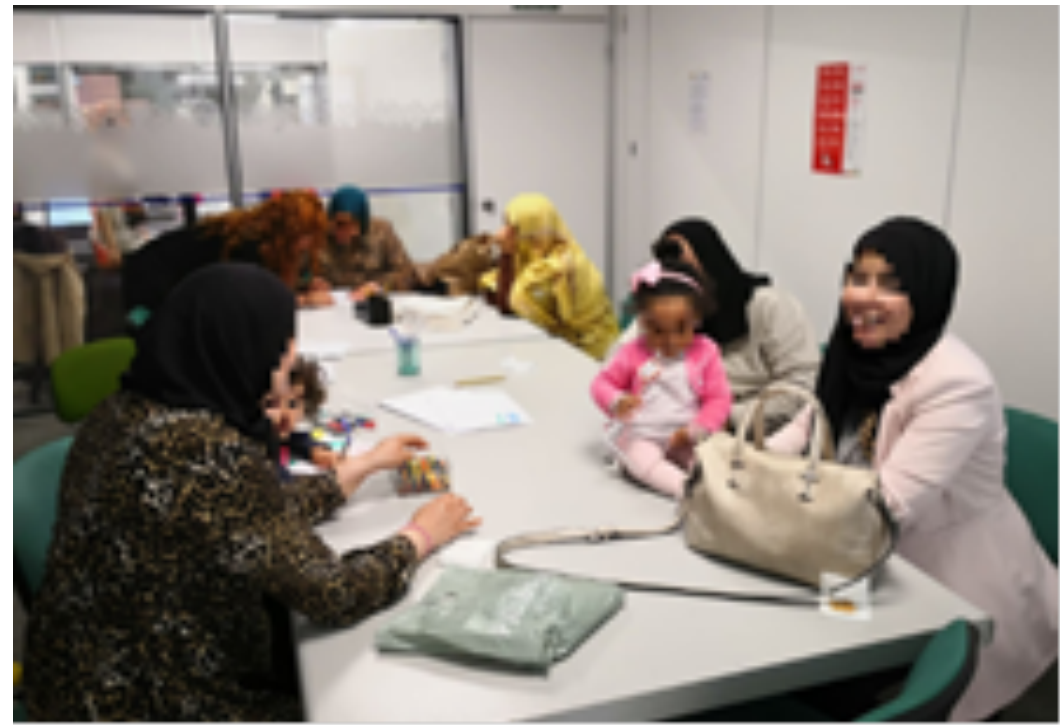

Imagen 2. Grupo de conciliación al que las alumnas acuden con los bebés a su cargo.

Es esta una de las actividades en las que la perspectiva de género cobra mayor relevancia. Se aprovecha la formación lingüística y sociocultural en un espacio entre iguales para hacer hincapié en la desigualdad sistemática en la vida cotidiana por cuestión de género, además de en los roles, funciones y responsabilidades femeninas. Se utiliza esta actividad para acompañar a las mujeres hacia mayores cotas de autonomía y participación en su entorno social, desde el respeto a la toma de decisiones de las propias mujeres.

Otra de las actividades exclusivamente presenciales son los talleres de formación para la mejora de la competencia digital. En forma de tutorías individualizadas o de talleres en 
grupos reducidos, el dominio de destrezas digitales requiere, al menos en sus inicios, la presencia física de un docente que guíe en todo el proceso.

La segunda de las modalidades de formación es la semipresencial o aprendizaje combinado (del inglés Blended Learning). Como su nombre indica, combina las sesiones en el aula con la tele-formación (García, Ferreira y Morales, 2011). Esas actividades sirven como refuerzo a las personas que están realizando la formación online y precisan de apoyo. Previamente y en función de la demanda, se organizan talleres de alfabetización sobre contenidos relacionados con el uso de Internet, la navegación por la Web y la práctica con la plataforma Moodle. Los apoyos presenciales se realizan en horario flexible en tutorías individuales principalmente, aunque en algunos casos se organizan talleres en grupos muy reducidos que permiten el desarrollo de aprendizaje cooperativo. Las sesiones presenciales se utilizan para la aclaración de dudas y la orientación del aprendizaje autónomo a través de Internet. Desde nuestra experiencia con los cursos online se observa que la mayor dificultad, en algunos casos, estriba en la carencia de competencia digital que, unida a falta de competencia lingüística, provoca el desánimo en las personas participantes si no existe contacto presencial. La formación semipresencial se orienta a suplir esa carencia.

La tercera de las modalidades acontece a distancia. En esta opción se llevan a cabo tres cursos de español como segunda lengua de diferente nivel y contenido; un curso online sobre contenidos relacionados con la Constitución y aspectos socioculturales de España $\mathrm{y}$ otro sobre el examen teórico del carnet de conducir y la seguridad vial. Sus características son las siguientes:

- El curso de formación online de español como segunda lengua de nivel inicial A1 tiene 150 horas de duración. Se desarrollan tres ediciones al año.

- El curso de formación online de español como segunda lengua de nivel preintermedio A2 dura un total de 80 horas y anualmente se ponen en marcha cinco ediciones.

- El curso de formación online de lengua y cultura españolas está enfocado a un nivel B1 de dominio del idioma y tiene contenidos especialmente relacionados con el conocimiento sociocultural de España. Cada año se realizan cinco ediciones de 60 horas (seis semanas) cada una.

- El curso online conocimiento constitucional y sociocultural de España, con 80 horas de duración, se corresponde, básicamente, con los contenidos cuya adquisición es necesaria para superar una de las pruebas indispensables para la obtención de la nacionalidad española. Cuenta con cinco ediciones cada año.

- El curso online prueba teórica del permiso de conducción, con 80 horas de duración, tiene como propósito favorecer el conocimiento de los contenidos obligatorios en materia de conducción para facilitar la obtención del permiso y sus correspondientes pruebas acreditativas. Al igual que los demás cursos de la misma extensión, son cinco las ediciones que se ofrecen de manera anual.

Cabe destacar que todos los cursos online de español como segunda lengua incluyen módulos formativos específicos, con contenidos que hemos denominado transversales y 
que tienen por objeto llevar la formación más allá de lo lingüístico. En todos ellos se combina la reflexión sobre los temas con los contenidos lingüísticos acordes con el nivel del grupo. Los módulos son:

- Perspectiva de género: se trabaja la igualdad entre hombres y mujeres.

- Sensibilización medioambiental: se profundiza en el cuidado del planeta y la conciencia medioambiental.

- Organización territorial y festividades de España: la atención se centra en el conocimiento del contexto, su organización, aspectos culturales, etc.

La formación online se corresponde con la idea de aprendizaje en cualquier momento y lugar, con un aprendizaje ubicuo (Burbules, 2014), en el que la responsabilidad del proceso recae en el alumno que decide dónde y cuándo aprender. Para poder ajustarse a la heterogeneidad del alumnado es necesario contar con herramientas intuitivas y sencillas de utilizar que se adapten a diferentes niveles de competencia digital. En nuestro caso, para los cursos a distancia se utiliza la plataforma Moodle ${ }^{3}$. Se trata de una herramienta de gran utilidad en el ámbito educativo, ya que permite tanto la gestión de cursos totalmente virtuales, como la utilización de un espacio en línea para dar apoyo a las clases presenciales. Las actividades de formación utilizan los recursos que ofrece la plataforma: tareas, consultas, foros, diario, cuestionarios, recursos, encuestas y wikis, además de otros recursos disponibles y sin olvidar el uso de las redes sociales como Facebook y Twitter.

La plataforma Siempre hay tiempo (imagen 3) fue creada en la primera edición del proyecto, en el año 2015, y se ha ido actualizando con el tiempo. Puede consultarse en el siguiente enlace: www.siemprehaytiempo.accem.es

\section{in Accem}

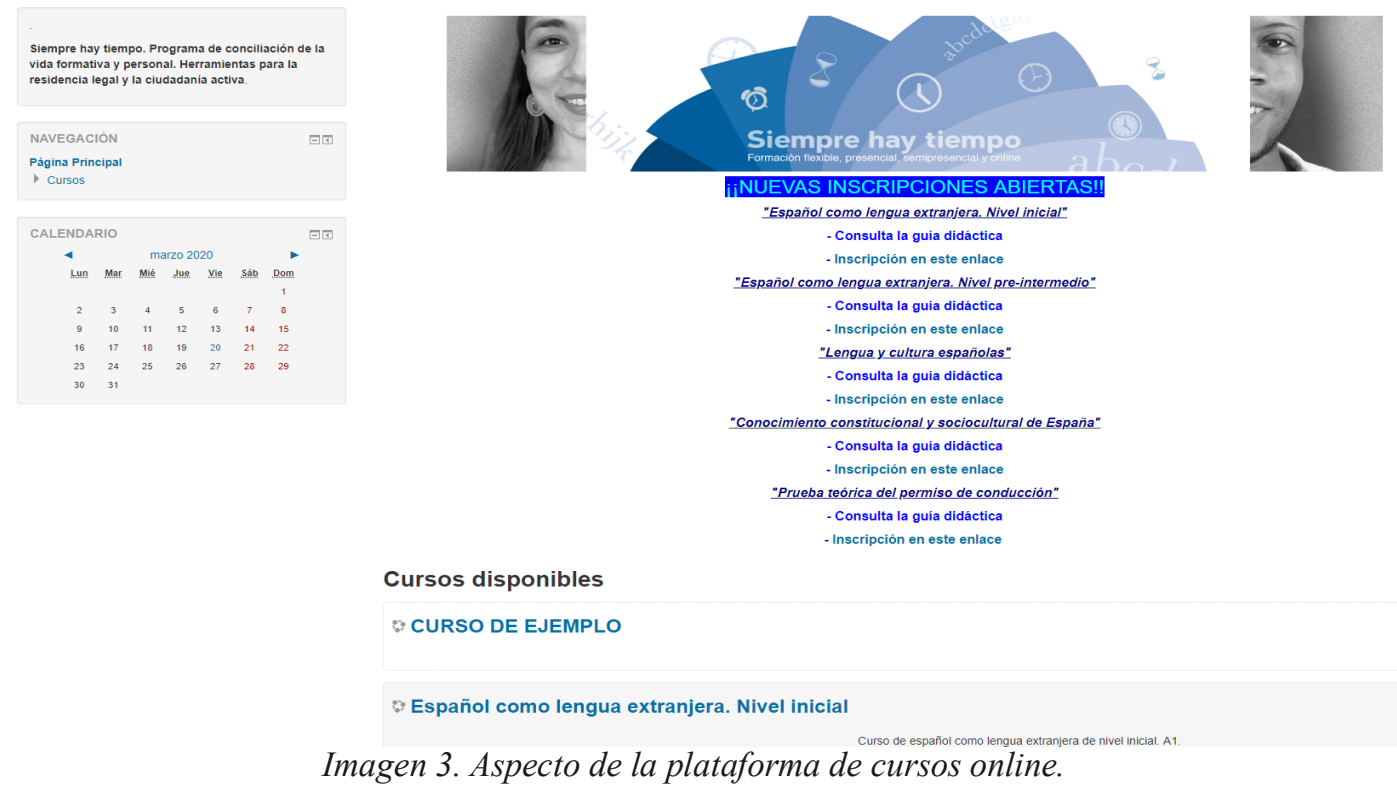

3 Moodle es una plataforma de aprendizaje diseñada para proporcionar a educadores, administradores y estudiantes un sistema integrado único, robusto y seguro para crear ambientes de aprendizaje. (htps://moodle.org). 
Es habitual que en la enseñanza del español a alumnado inmigrante las actividades se orienten a la práctica de destrezas orales (Instituto Cervantes, 2005). La urgencia del aprendizaje para estos estudiantes apunta a una enseñanza encaminada a fines laborales y sociales donde se privilegia la comunicación oral inmediata y la escritura suele verse reducida a intercambios prácticos relacionados con los trámites formales $\mathrm{o}$ administrativos. Los cursos a distancia pretenden compensar esa falta de atención y en ellos predominan las tareas de comprensión y expresión escritas. Los cuestionarios abiertos, los foros, los chats, las lecciones, los paquetes de contenido, etc. se dirigen primordialmente a este objetivo. En el marco de una plataforma educativa, además, encajan los usos de la escritura electrónica que van desplazando paulatinamente al lápiz y el papel. Las actividades dentro de la plataforma estimulan la creatividad, se convierten en interacciones reales en las que el resto de aprendices y docentes participan. Permiten intercambiar puntos de vista, compartir experiencias y ofrecen audiencias y contextos reales con respuesta. Es en este tipo de enseñanza donde más se pone en evidencia lo multimodal (Cassany, 2003). A la escritura alfabética le acompañan fotos, colores, gráficos, iconos (emoticonos) en diferentes géneros digitales como el correo electrónico o el foro (imagen 4) y se utiliza la escritura para interactuar, pero también para aprender la segunda lengua.

\section{is Accem}
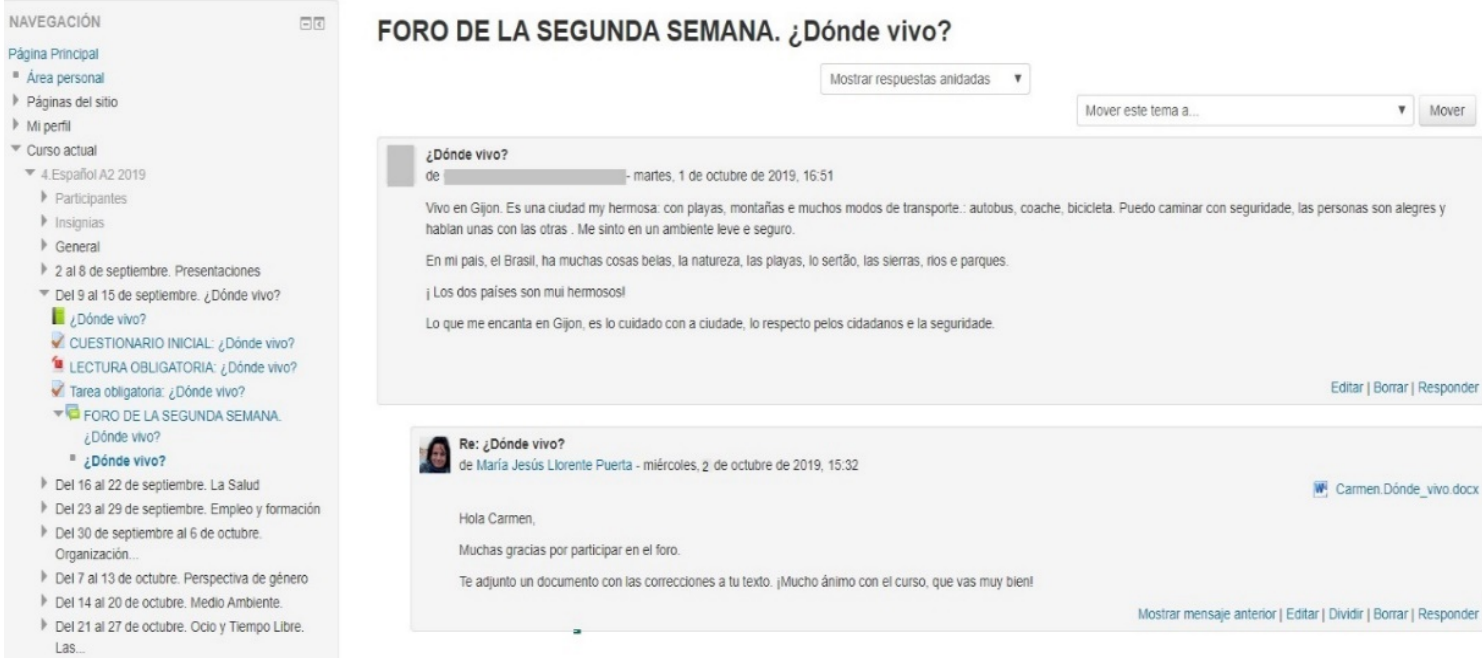

Imagen 4. Ejemplo de uno de los foros de un curso.

El aprendiz recibe una retroalimentación grupal a través de los foros e individualizada por medio del correo electrónico. Se envía a cada estudiante que lo precisa una corrección de sus errores gramaticales o léxicos con la explicación de la forma correcta y se le facilitan enlaces externos a páginas o sitios web donde pueden realizar actividades complementarias de refuerzo de atención a la forma.

La plataforma permite que el propio alumno pueda comprobar la adecuación de sus respuestas y autoevaluar sus conocimientos. No obstante, la figura del docente resulta fundamental para garantizar la interacción, proporcionar retroalimentación y, sobre todo, estimular la participación y mantener el interés y la motivación hacia los cursos. Para la evaluación del proyecto se realiza, por un lado, un seguimiento del alumnado que incluye tres fases: 
En la formación presencial, se lleva a cabo una entrevista individual inicial que cumple la doble función de detectar necesidades y diagnosticar el nivel de dominico de la segunda lengua.

- Una evaluación continua mediante tutorías individualizadas y grupales, y fichas de seguimiento de la formación.

- Una evaluación sumativa en la que se hace una valoración global del grado de participación de los estudiantes, las estrategias digitales y comunicativas que emplean, y el nivel de autonomía que han adquirido como aprendices. Según los diferentes cursos y talleres se realizan de forma complementaria una serie de actividades obligatorias y trabajos finales e incluso, algunos cursos incluyen pruebas objetivas.

Por otro lado, para indagar acerca del funcionamiento del programa, las personas participantes, al finalizar los talleres y cursos, realizan encuestas o entrevistas de valoración.

En todas las formaciones, tanto presenciales como a distancia, se solicita al alumnado que realice una tarea final que consiste en elaborar un texto en el que se refleje su aprendizaje, su opinión acerca de los aciertos y los fallos de los cursos y toda sugerencia que considere oportuna. Mediante este trabajo, al tiempo que se lleva a cabo una autoreflexión sobre el propio proceso de aprendizaje, el profesorado obtiene información sobre la eficacia del curso y saca ideas para la mejora. Estas actividades generan una evaluación realmente significativa que produce textos como el siguiente de una alumna del curso de lengua y cultura españolas que valora tanto el aprendizaje sociocultural como el gramatical:

Tarea final

Yo gusté mucho del curso. Yo aprendí la historia de España, me ha gustado conozer muchos lugares llamados los mejores de España: Granada, Alhambra, Sierra Nevada, Murcia, pueblos de la provincia de Murcia, Alicante, Bullas, entre otros. Valencia, Barcelona con su parque Güel, La Sagrada Familia, la Ranbla lanbra e su mercado, sus cantantes, sus bailarines guapísimos. He estado en Madrid, ciudad histórica encantadora. Picos de Europa e sus pueblos, parque y naturaleza. Galicia es rica en gastronomía. Las playas de España son diferentes de las Brasileñas, son montañosas, con naturaleza de un verde mui especial.

También aprendi mucho acerca los pronombres. Ha sido mui importante en mi aprendizado de Español. Soy agradecida a las maestras de Accem e las muchas oportunidades que ha me proporcionado.

Gracias

\section{Algunos datos}

Presentamos, para finalizar, un resumen de los hitos cuantitativos del proyecto a lo largo de este lustro de andadura:

- Siempre Hay Tiempo comenzó en el año 2015 con un único curso piloto online. En el curso online, del que se celebraron cuatro ediciones, se inscribieron 137 personas y finalmente llegaron a participar 59. En todo el proyecto fueron 150 los alumnos (66 hombres y 84 mujeres).

- Al año siguiente se amplió la oferta online. Al curso de español, del que se llevaron a cabo cinco ediciones, se le sumaron un curso de lengua y cultura 
españolas y uno de conocimiento constitucional y sociocultural de España que obtuvo 141 matrículas en sus cinco ediciones.

- En el año 2017 fueron 91 hombres y 122 los participantes en el proyecto (un total de 213 personas). La novedad esa convocatoria fue un curso de ELE de nivel inicial online, con una duración de 150 horas a lo largo de 15 semanas, del que se realizaron tres ediciones y que tuvo 34 matrículas.

- Los cursos 2017 y 2018 son los de consolidación del proyecto: 255 participaron en 2018. Las cinco ediciones del curso de español tuvieron 136 matrículas, las del curso de conocimiento constitucional y sociocultural de España, 132 matrículas, las cuatro del curso de lengua y cultura españolas, 88 inscripciones y las tres del curso de español de nivel inicial 115 matrículas. Como novedad, en 2018 se puso en marcha un curso online para ayudar a preparar el examen teórico del carnet de conducir que obtuvo 158 matrículas a lo largo de sus cinco ediciones.

- El año 2019 fue el de afianzamiento del programa. En él no se ofreció ningún curso nuevo, pero participaron en su formación 284 personas diferentes y se inscribieron más de 600 personas en los cursos online.

\section{Conclusiones.}

Consideramos que la formación online es uno de los mayores éxitos del programa. Consideramos que la formación online es uno de los mayores éxitos del programa. A pesar de que el e-learning cuenta con un gran número de ventajas, según Fernández Jiménez (2015) uno de los problemas principales que presenta es el de un mayor índice de abandono de estudiantes si lo comparamos con otras modalidades de formación. En nuestro caso, destacamos que el porcentaje de finalización de los cursos online llega aproximadamente al $50 \%$ de las personas que se matriculan, un porcentaje que se corresponde con el habitual en las formaciones a distancia.

Por otra parte, las tutorías individuales de alfabetización digital dan respuesta a las necesidades de asesoramiento educativo manifestadas por las personas participantes en los diversos contextos de formación y sen rebelado como un modelo de práctica exitosa.

El trabajo transversal y también las actividades específicas, como los grupos de conciliación a los que acuden mujeres acompañadas de los menores suponen mucho más que una actividad formativa.

El proyecto Siempre Hay Tiempo intenta ajustarse a las necesidades de las personas migrantes que tienen dificultades de acceso a la formación lingüística en el nuevo idioma, desconocen aspectos socioculturales básicos de su entorno y que, además, tienen una competencia digital emergente. Este programa evidencia la necesidad de una figura docente multitarea capaz de poner en práctica una enseñanza que atienda a las necesidades e intereses de personas de todas las edades y procedencias, mediante canales diversos, referencias múltiples y opciones didácticas enfocadas a estilos de aprendizaje particulares.

Con el apoyo de una enseñanza más allá de la formación lingüística, con una dimensión intergeneracional, que combina la modalidad presencial, semipresencial y a distancia y que atiende a la dimensión intercultural se ha conseguido que personas que quedaban 
fuera de los ámbitos formativos tradicionales puedan recibir una formación necesaria para una integración efectiva en la sociedad de acogida como ciudadanos y ciudadanas conscientes de sus derechos y deberes.

\section{Bibliografía.}

BERRIOS BARRA, L. (2018). Una experiencia didáctica a partir del hipertexto digital y la multimodalidad con profesores de Lengua y Literatura: aproximación de los clásicos a los adolescentes. Tesis doctoral dirigida por Margarida Prats Ripoll y Antonio Mendoza Filloa (dir. Tes.). Barcelona: Universidad de Barcelona.

BURBUles, N. (2014). «Aprendizaje ubicuo: nuevos contextos, nuevos procesos», Revista Entramados-Educación y sociedad, (1), pp. 131-135.

CASSANY, D. (2003). La escritura electrónica. Recuperado el 31/08/2020 de: http://www.redined.mec.es/oai/indexg.php?registro $=008200430558$.

EsteBAN GUITART, M. (2011). «Aplicaciones contemporáneas de la teoría vygostkiana en educación», Revista Educación y Desarrollo Social, 5(1), pp. 95-113.

FERNÁNDEZ JiMÉNEZ, M.A. (2015). Tutoría en e-learning. Funciones y roles del tutor en la formación online. Tesis doctoral dirigida por Juan Carlos Tójar Hurtado y Esther Mena Rodríguez (dir.tes.). Málaga: Universidad de Málaga.

García salinas J., Ferreira CABrera, A. y Morales Ríos, S. (2011). «Autonomía en el aprendizaje de lenguas extranjeras en contextos de enseñanza mediatizados por la tecnología», Onomázein, 25 (1), pp. 15-50.

GARDNER, R.C. y LAMBERT, W.E. (1972). Attitudes and Motivation in Second Language Learning. Mass: Newbury House Publishers.

Instituto Cervantes. (2005). Español como nueva lengua. Orientaciones para un curso de emergencia destinado a inmigrantes adultos. Instituto Cervantes y Anaya. Recuperado el 31/08/2020 de: https:/cvc.cervantes.es/ensenanza/biblioteca_ele/inmigracion/orientaciones/default.htm

Instituto Cervantes. (2006). Plan curricular del Instituto Cervantes. Niveles de Referencia para el español. Biblioteca Nueva. Versión electrónica en: https://cvc.cervantes.es/ensenanza/biblioteca_ele/plan_curricular/default.htm

INSTITUTO CERVANTES. (2012). Las competencias clave del profesorado de lenguas segundas $y$ extranjeras. Recuperado el 31/08/2020 de: http://cvc.cervantes.es/ensenanza/biblioteca_ele/competencias/default.htm

Instituto Cervantes. (2006). Plan curricular del Instituto Cervantes. Niveles de Referencia para el español. Biblioteca Nueva. Versión electrónica en: https://cvc.cervantes.es/ensenanza/biblioteca_ele/plan_curricular/default.htm

INTEF. (2017). Marco Común de Competencia Digital Docente. Madrid: Ministerio de Educación, Cultura y Deporte. Recuperado el 31/08/2020 de: https://intef.es/material_seg/marco-de-competencia-digital/

KRESS, G. (2010). Multimodality. A social semiotic approach to contemporary communication. London: Taylor \& Francis. 
Llorente Puerta, M.J. (2018). «25 años del español (L2/LE) para inmigrantes», Boletín de la Asociación para la Enseñanza del Español como Lengua Extranjera (ASELE), 58, pp. 43-65.

Maslow, A. (2013 [1943]). A theory for Human Motivation. España: Martino Fine Books.

PAstor Cesteros, S. (2007). «Enseñar una segunda lengua (SL) y en una SL: hacia una nueva formación del profesorado de español para inmigrantes». Linred (Lingüística en la red). Monográfico: La Enseñanza de Segundas Lenguas a Inmigrantes. 5, Anexo 1 .

Richards, JACK C. y Rogers, TheOdore S. (2002). Enfoques y métodos en la enseñanza de idiomas. Cambridge: Cambridge University Press.

Ríos RoJAS, A. (2016). «La inmersión como experiencia de aprendizaje y su efecto en el aula de español como nueva lengua para inmigrantes» en G. Ruiz Fajardo y A. Ríos Rojas (eds.). Inmigración: nuevas lenguas, nuevos hablantes, nuevas perspectivas. (pp. 13- 31). Sevilla: Universidad internacional de Andalucía.

SANZ, F.I. (2005). Español como nueva lengua. Orientaciones para un curso de emergencia destinado a inmigrantes adultos. Instituto Cervantes y Santillana. Universidad de Salamanca. Recuperado el 31/08/2020 de: https://cvc.cervantes.es/ensenanza/biblioteca_ele/inmigracion/orientaciones/cvc_ecnl.pd $\mathrm{f}$

Villalba Martínez, F. y Hernández García, M.T. (2010). «Perspectivas y líneas de trabajo en la enseñanza del español a inmigrantes». En Miquel, L. y Sans, N. (coord.), Didáctica del español como lengua extranjera. Cuadernos de Tiempo Libre. Colección Expolingua. Madrid, Fundación Actilibre (pp. 163-184). Reeditado por MarcoEle, Revista de Didáctica del Español como Lengua Extranjera, Monográfico, núm. 10.

VillalBa MARTínez, F. (2012). El discurso expositivo del profesor en contexto escolar en L1 y L2. Tesis doctoral dirigida por Isabel García Parejo. (Dir. Tes.). Madrid: Universidad Complutense de Madrid.

Woodward, T. (2002). Planificación de clases y cursos. Cambridge: Cambridge University Press. 